\title{
L’association Natures Sciences Sociétés-Dialogues crée une antenne à Montpellier
}

L'association Natures Sciences Sociétés-Dialogues (NSS-D) a développé à Montpellier une relation privilégiée avec les établissements de recherche et d'enseignement supérieur (Cirad, CNRS, IRD, Irstea, Ifremer, Universités, Agropolis International). En témoigne l'organisation de 2003 à 2006 d'un atelier sur la modélisation de l'environnement et, en 2005, d'un colloque qui a donné lieu à la parution d'un ouvrage ${ }^{1}$ en 2009.

La communauté scientifique locale est très attachée à traiter des interfaces société-environnement et revendique une spécificité en s'intéressant aux enjeux de développement. Ce positionnement s'est récemment traduit par la tenue à Montpellier de plusieurs conférences internationales (sur la résilience, l'ethnobotanique, l'irrigation et le drainage, l'écologie, l'agriculture familiale, la climate smart agriculture ${ }^{2} . .$. ) mais également, à l'échelle régionale, par des regroupements d'unités de recherche à vocation interdisciplinaire large ainsi que par la mise en place de formations interdisciplinaires ${ }^{3}$.

Dans toutes ces manifestations scientifiques ou institutionnelles, l'enjeu est de comprendre les conditions d'évolution des sociétés en relation avec les milieux et les ressources naturelles présentes dans leurs environnements, ces ressources - qu'elles soient consommées ou protégées -, étant parties prenantes des trajectoires sociales, économiques et politiques. Rejoignant en cela le projet de NSS-D, dans lequel elle privilégie les problèmes du développement, cette communauté scientifique montpelliéraine se consacre aux questions des

\footnotetext{
1 Hervé D., Laloë, F. (Eds), 2009. Modélisation de l'environnement : entre natures et sociétés, Versailles, Quæ-NSS-Dialogues.

2 Par exemple, la conférence Resilience 2014 « Resilience and development: mobilizing for transformation » organisée par le réseau Resilience Alliance (Montpellier, 4-8 mai 2014, http:/ / www.resilience2014.org) ou la conférence «Climate smart agriculture 2015» (Montpellier, 16-18 mars 2015, http:/ / csa2015.cirad.fr).

3 Cf. le site internet d'Agropolis International: http:// www.agropolis.fr/formation.
}

transformations sociales et environnementales, des états de référence à partir desquels ces transformations peuvent être perçues, des modalités politiques de construction de ces états de référence et des projets de société qui les sous-tendent.

Les approches scientifiques mises en œuvre pour aborder ces questions associent, dans un pluralisme disciplinaire, divers objets, terrains et méthodes. La modélisation en fait partie, mais elle ne peut suffire sans l'apport de l'observation de long terme des changements sociaux et environnementaux, ni sans celui des analyses de documents témoignant de l'évolution des sociétés. Développer le partage de connaissances et d'expériences entre les équipes impliquées dans la compréhension de ces dynamiques doit permettre de renforcer le savoirfaire propre à l'interface entre disciplines mais aussi de faire émerger les liens orphelins entre secteurs de recherche. C'est dans cet objectif qu'une antenne de NSS-D est créée à Montpellier afin de favoriser rencontres, échanges, collaborations entre chercheurs des différents domaines et disciplines ayant pour objet les rapports entre natures et sociétés.

Faire d'une telle antenne un lieu d'animation offre une originalité au niveau national voire international en raison de la configuration de la communauté montpelliéraine. Elle regroupe en effet des chercheurs en sciences de l'information, en sciences agronomiques, en sciences de l'environnement (écologie, eau, biodiversité) ainsi qu'en sciences économiques, humaines et sociales, travaillant tous sur les milieux continentaux, littoraux et marins en région, mais aussi en Méditerranée et dans les Suds. Elle bénéficie de la présence de structures fédératives telles qu'Agropolis International ou la Maison des sciences de l'homme de Montpellier (MSH-M), autant d'institutions facilitant la mise en œuvre et la bonne synergie de ces collaborations. Dans son nouveau projet de création d'une fédération de recherche, la MSH-M souhaite par exemple développer une identité scientifique reposant sur les forces vives du site, en cohérence avec les politiques nationales et européennes de la 
recherche. Son ambition est de devenir un des principaux pôles de recherche en sciences sociales contribuant à l'analyse des relations entre l'environnement et le développement, avec un axe privilégiant la qualité de vie. Pour ce faire, elle prévoit de renforcer la structuration des différentes communautés scientifiques régionales et d'œuvrer, sur le site et en dehors de celui-ci, à la construction d'échanges scientifiques, en particulier Nord/Sud, et de formations, ainsi qu'à l'articulation des SHS et des autres sciences. Autant d'éléments qui renforcent la pertinence et la cohérence de l'initiative de NSS-Dialogues.

Plus concrètement, la création de l'antenne montpelliéraine par l'association NSS-D se traduira dans les activités suivantes :

- animer, dès 2016, un séminaire régulier trimestriel présentant des recherches aux interfaces société-environnement, chaque séance, afin de favoriser le débat, comprenant les interventions d'un jeune chercheur de la communauté montpelliéraine et d'un chercheur invité ;

- labelliser et rendre visibles dans un ensemble cohérent des conférences de collègues étrangers de passage ou en accueil dans les unités ${ }^{4}$;

- promouvoir la formation à l'interdisciplinarité dans les formations universitaires et les écoles d'ingénieurs.
Ces activités répondent ainsi à deux enjeux principaux pour la communauté montpelliéraine. Un enjeu conceptuel, car c'est en convoquant les concepts de résilience et d'adaptation qu'elle aborde l'interface sociétéenvironnement sous l'angle des transformations, innovations et changements, dans un contexte en évolution constante et rapide. Un enjeu de formation à la recherche, $\mathrm{d}^{\prime}$ où le choix d'associer, dans le comité d'organisation du séminaire, des doctorants des Écoles doctorales rassemblant les disciplines impliquées dans cette démarche, afin d'évaluer avec eux les apports de chaque séance et de proposer à moyen terme un module doctoral qui corresponde aux attentes des étudiants et aux thématiques émergentes.

Outre la conception de ce module doctoral, la dynamique ainsi générée a également vocation à susciter des textes pour les pages de la revue Natures Sciences Sociétés ou du site web de l'association ${ }^{5}$.

Une information régulière qui n'exempte pas toute la communauté de la revue NSS et de la collection « Indisciplines » de venir débattre à Montpellier même.

Olivier Barreteau, Dominique Hervé, Francis Laloë, Bernard Moizo
$4 \quad$ Plus de dix unités de recherche ont déjà témoigné de leur intérêt pour cette initiative.

\footnotetext{
5 http://www.nss-dialogues.fr.
} 